\title{
Multi-Radio Cooperative ARQ in Wireless Cellular Networks: A MAC Layer Perspective
}

\author{
J. Alonso-Zárate ${ }^{1}$, E. Kartsakli2 ${ }^{2}$ L. Alonso ${ }^{2}$, M. Katz ${ }^{3}$ and Ch. Verikoukis ${ }^{1}$ \\ ${ }^{1}$ Centre Tecnològic de Telecomunicacions de Catalunya (CTTC) \\ Av. Carl Friedrich Gauss 7, CTTC, 08860 Castelldefels, Barcelona (Spain) \\ E-mail: [jesus.alonso, cveri]@cttc.es \\ 2Department of Signal Theory and Communications, \\ Universitat Politècnica de Catalunya (UPC) \\ Av. Esteve Terradas, 7, EPSC, Campus UPC, 08860 Castelldefels, Barcelona (Spain) \\ E-mail: [ellik, luisg]@tsc.upc.edu \\ ${ }^{3}$ University of Oulu, Finland \\ E-mail: marcos.katz@ee.oulu.fi
}

\begin{abstract}
Multi-Radio Cooperative Automatic Retransmission Request (MCARQ) schemes are introduced in this paper within the context of hybrid networks which combine long-range and short-range communications. Since the number of wireless devices is incessantly increasing, it is frequently possible to establish a spontaneous cooperative cluster in the close proximity of any wireless device. These devices forming the cluster are connected to both a cellular-based network such as WiMAX, 3G, or LTE and a short-range network based on technologies such as WLAN, Zigbee, Bluetooh, or UWB, among other possibilities. The main idea behind the proposed MC-ARQ scheme is that, upon transmission error through the cellular interface, retransmission can be requested to the wireless grid surrounding the destination device using the short-range interface instead of the primary cellular link. Therefore, besides the cooperative diversity attained with CARQ schemes, the traffic load in the cellular interface is reduced benefiting thus a high number of users and reducing both energy consumption and interference. The Persistent Relay Carrier Sensing Medium Access (PRCSMA) protocol is presented as an example of solution for the MAC layer in this emerging new topic.
\end{abstract}

Keywords: cooperative communications; heterogeneous networks; cooperative ARQ; Medium Access Control (MAC) 


\section{Introduction}

Although the concept of cooperation in wireless communication networks was first presented almost four decades ago [1], the seminal works of Sendonaris et al. [2] and Laneman [3] triggered a vast amount of research in the topic since 2000. Cooperative communications is still today a very popular and highly diversified research topic [4]. Most of the research efforts so far have been focused on fundamental and theoretical studies, while less work has been devoted to developing concrete practical applications of cooperation. Most of the early work on cooperative communications has focused on improving transmission parameters at the physical layer. However, the truly emergence of cooperative communications needs to be supported by extensive research on higher layers of the protocol stack as well as the development of novel business models. We deal in this paper with a simple and practical way of applying cooperation in the real world; wireless grids [5]. A wireless grid is a cooperative spontaneous cluster made of heterogeneous wireless devices in close proximity of each other. These devices are connected to a conventional infrastructurebased cellular network, e.g., to an Access Point (AP) or a Base Station (BS), and they are also connected to each other through short-range links. GSM/GPRS, 3G, LTE, WiMAX or satellite are representative examples of wide area cellular access, while short-range connectivity can be provided by technologies such as Wireless Local Area Network (WLAN), Zigbee, Bluetooth, or UWB among other possibilities. Therefore, a hybrid communication architecture combining centralized and distributed access topologies is considered. Such composite communication architecture, a widely unexplored research field, can benefit the whole value chain, from network, service and content providers, to manufactures as well as the end-users. One of the keys for the success of the wireless grid concept is to develop cooperative strategies for which all interacting devices (or users behind them) will get some advantage, resulting in a natural incentive for users to cooperate.

According to visions of the Wireless World Research Forum (WWRF), by the year 2017, seven trillion wireless devices will be serving seven billion people. Clearly, most of these devices will combine both cellular and short-range communication interfaces. One of the main consequences of these figures is that there will be always a potential cooperative cluster surrounding any wireless device regardless of its specific location. In fact, this is a reasonable assumption even today. It is possible to interconnect a laptop, a mobile phone, and a PDA to create a Personal Area Network (PAN), having thus an already deployed personal wireless grid. This can be extended to office and home environments, where the number of wireless devices is increasing day by day. Although more 
complex to manage, the wide urban environment could also provide wireless grid availability. This classification is illustrated in Figure 1.

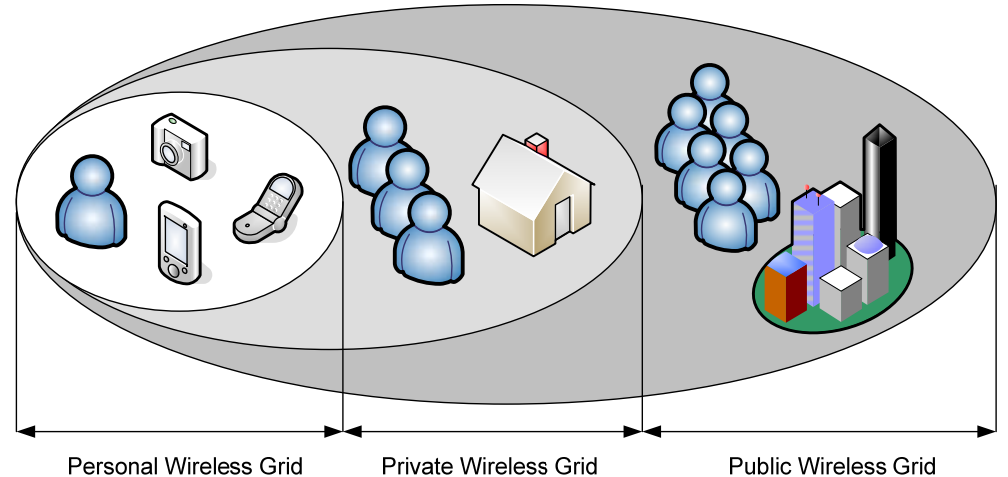

Figure 1 Different operating scenarios for wireless grids

It is worth emphasizing that the wireless grid concept can be in principle implemented with existing technologies, as many commercial wireless devices today integrate onboard multiple air interfaces. In fact, this trend is expected to continue and strengthen in the future. However, so far, a given interface is typically used for a particular application. The concept of wireless grids exploits interoperation of these technologies, as illustrated in Figure 2.

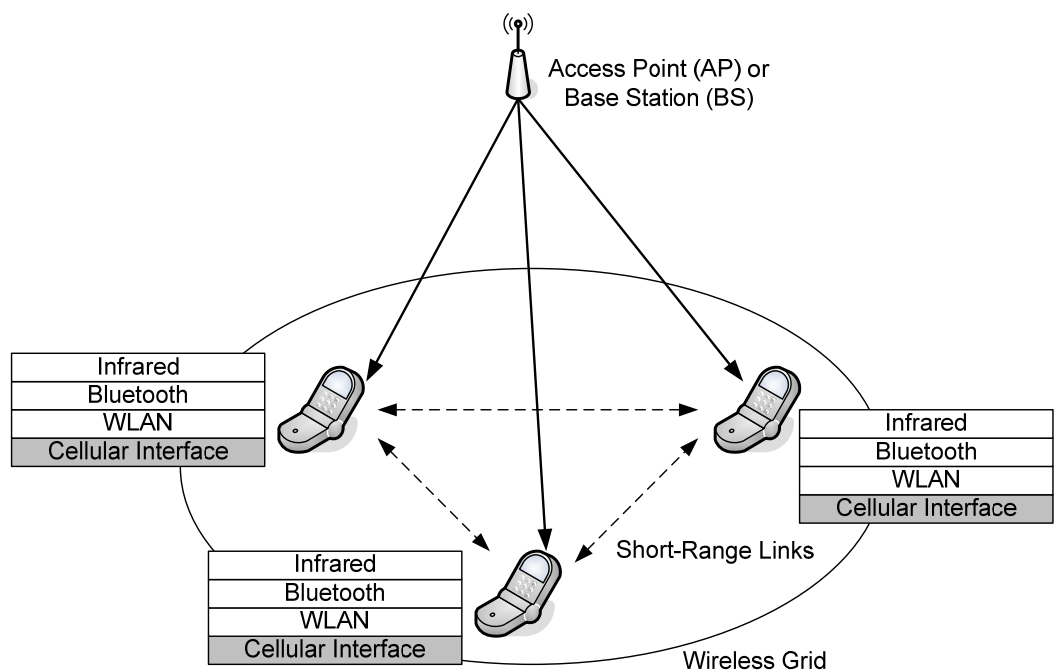

Figure 2 Wireless Grid: composite network with infrastructure-based networks and peer-to-peer communications 
It is worth emphasizing that this concept is different from that of vertical handover, which represents the change of radio interface for seamless communications. In our case, we deal with the interoperation of the different interfaces simultaneously. Note that wireless grids can help in distributing information from the cellular (infrastructure-based) network to the customers in order to improve energy efficiency of wireless devices, to increase network throughput, to increase delivery reliability or to reduce interference to other systems. However, in order to bring cooperation in wireless grids to life, it is still necessary to study and analyze their operation from a wide range of points of view. In fact, the wireless grid concept opens an enormous vast of communication opportunities that can be exploited in several ways.

We present in this paper the concept of the Multi-Radio Cooperative Automatic Retransmission Request (MC-ARQ) scheme at the Data Link Layer (DLC) of wireless grid systems. The main idea is to execute a Cooperative ARQ (C-ARQ) scheme while exploiting the multi-radio capabilities of wireless grids. Since the first contribution of Zimmermann et al. related to C-ARQ in wireless networks [6], a variety of C-ARQ schemes have been proposed as a solution to combat the wireless channel fading and improve the performance of traditional (non-cooperative) ARQ schemes in wireless networks. We will review the main contributions later in Section 4. C-ARQ schemes exploit the broadcast nature of the wireless channel by which a transmission can be heard not only by the intended destination but also by any device in the transmission range of the transmitter. What has been traditionally considered as interference, is used in CARQ schemes to provide alternative uncorrelated retransmission paths upon error occurrence, i.e., spatial diversity. The devices which overheard the original transmission can act as spontaneous helpers, partners, or relays and retransmit a copy of the original packet. This avoids a (probably costly in terms of radio resources) retransmission from the source through the same channel where the error has occurred, which might remain in bad conditions for some time due to the space-time correlation of the wireless channel. The MC-ARQ scheme presented in this paper extends this idea to wireless grids with multiple radio interfaces available at each device. Upon the reception of a data packet with errors within the cellular interface, retransmissions can be requested to any of the potential local helpers, i.e., any of the users forming the wireless grids. However, unlike in C-ARQ schemes, these retransmissions can be performed through a different interface, i.e., any of the short-range wireless interfaces available both at destination and at the helpers. Therefore, the MC-ARQ scheme attains:

1. The cooperative diversity gains of C-ARQ schemes as the helpers provide independent transmission paths.

2. The implicit benefits of using short-range communications, i.e., lower transmission power and thus lower energy consumption and interference.

3. The unloading of the cellular link. This is probably the most important achievement since yields a benefit for the whole network and thus can be perceived by the helpers as an incentive to cooperate. 
In this paper we analyze the unique characteristics of MC-ARQ schemes from the MAC layer point of view and identify future challenges to be investigated. It should be taken into account that getting a number of devices involved in the communication process requires coordination, which is not costless. We also present the Persistent Relay Carrier Sensing Multiple Access (PRCSMA) protocol, previously described and analyzed in [7] and [8] for plain C-ARQ, as an example of MAC protocol suitable for the execution of the MC-ARQ. It considers that devices in the wireless grid (short-range) can get connected through a CSMA-based interface, such as WLAN 802.11 [9] or Wireless Sensor Networks 802.15.4 [10] based systems. A case study is briefly introduced in this paper to show the improved performance that can be attained in a composite cellular/WLAN network.

The remainder of the paper is organized as follows. In Section 2 we describe the proposed MC-ARQ scheme in the context of wireless grids. In Section 3 we discuss the unique challenges that such schemes pose to the design of the MAC layer. In Section 4 we review the state of the art in the design of cooperative MAC protocols and in Section 5 we identify the PRCSMA as an IEEE 802.11based MAC protocol suitable for the execution of a MC-ARQ scheme. Some simulation results are discussed in Section 6 and, finally, Section 7 concludes the paper and gives some final remarks.

\section{Multi-Radio Cooperative ARQ (MC-ARQ) in Wireless Grids}

We consider an infrastructure-based wireless network with a number of wireless devices associated to the cellular-based AP or BS. The terminals are equipped with additional wireless short-range interfaces that enable peer-to-peer communications. Due to the dynamic conditions of the wireless channel within the cellular interface some packets may be received at the destination with unrecoverable errors. These transmission errors can be detected at destination by attaching, for example, a Cyclic Redundancy Code (CRC) to the header of data packets. Due to the broadcast nature of the wireless channel, these transmissions can be overheard by the devices forming the wireless grid around the destination device, likely through independent and uncorrelated transmission paths. Therefore, upon the occurrence of a transmission error, retransmission can be requested either from the source through the cellular interface or, locally from one or more devices in the wireless grid. In the latter case, spatial diversity gains can be attained. The basic concept of cooperative diversity is illustrated in Figure 3. The proposed cooperative scheme can benefit both the involved nodes and the whole network in several aspects. In the case that retransmission is requested at the local level, the signal to interference ratio between neighbor cells is reduced with a consequence to the overall capacity of the network. Moreover, the available bandwidth of the cellular network can be used for another 
transmission and the total throughput of the cellular network may be increased (as it is freed from retransmitted traffic). In addition, the local short-range retransmission can be done at higher transmission rates and with lower energy consumption profiles. Therefore, the key of the MC-ARQ scheme is to exploit this spatial diversity and to span it to the frequency and technology domains by executing retransmissions through different wireless interfaces.

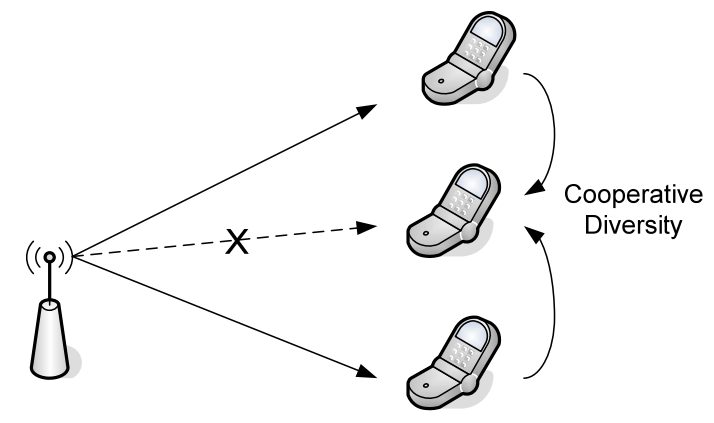

Figure 3 Cooperative Diversity

The MC-ARQ works as follows: all the devices listen to every ongoing transmission in the cellular interface in order to be ready to cooperate when required. In addition, they keep a copy of any transmitted data packet from the AP or BS (regardless of its destination address) until it is acknowledged by its intended destination. The copy retained by the devices might be stored at each device data buffer or in a different dedicated queue. Scheduling tasks are out of the scope of this paper, but they should be revisited to match the MC-ARQ operation. Whenever a destination receives a data packet with unrecoverable errors through the cellular interface, it broadcasts a retransmission request in the form of a control packet through a short-range wireless interface. A cooperation phase is then initiated. The control packet is referred to as the Call for Cooperation (CFC) packet. A set of the devices within the neighborhood of the destination which overheard the original transmission from the source and receives the CFC from the destination become active helpers and form the concept of wireless grid. Each of these active helpers attempts to retransmit a copy of the original packet to assist in the failed communication. Therefore, the requested retransmissions take place using a short-range technology and thus the main cellular link is unloaded. Although these retransmissions could be performed orthogonally in either time (TDMA), frequency (OFDMA), or code (CDMA), we focus hereafter on time-orthogonal retransmissions, which might be the simplest approach to implement. Eventually, the destination station might either receive an errorless copy of the original data packet or be able to properly combine the different retransmissions from the helpers to successfully decode the original packet. This event indicates the end of the cooperation phase and is notified to the wireless grid through the broadcast of an ACK packet. 


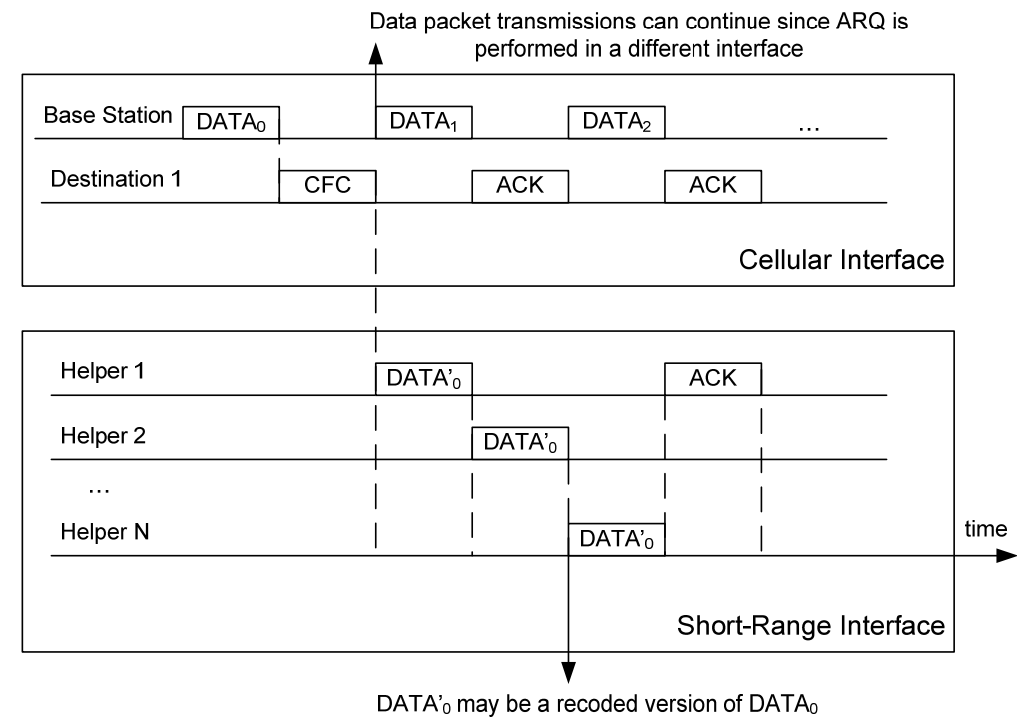

Figure 4 Multi-Radio Cooperative ARQ scheme

The ideal operation of the MC-ARQ scheme is exemplified in Figure 4 where the communication between a BS and a destination device is assisted by an arbitrary number of helpers. As shown in the picture, the most remarkable benefit derived from the execution of the MC-ARQ scheme is that data packet transmissions in the cellular interface can continue despite the occasional occurrence of transmissions errors. Recall that the MC-ARQ scheme is executed in a different and independent short-range wireless interface, and thus the occupation of the cellular interface for retransmissions is reduced.

The performance of this kind of MC-ARQ schemes is strongly influenced by the following factors:

1. The helper selection criteria: The CFC transmitted by the destination may attach some helper selection criteria. For example, it could attach a minimum required Signal to Noise Ratio (SNR) threshold in the reception of either the original data packet or the CFC packet in order to become an active helper. Although the helper selection problem is a very interesting topic itself, it is out of the scope of this paper.

2. The PHY forwarding technique executed by the active helpers: according to the specific strategy applied by the helpers it is possible to classify cooperative (helper) techniques as:

a. Amplify and forward techniques, when the helpers transmit an amplified version of the original signal. 
b. Compress and forward techniques, when the helpers send a compressed version of the original transmitted signal.

c. Decode and forward techniques, when the helpers transmit recoded copies of the original message. It has to be noted that using decode and forward, the recoding process can be done on the basis of repeating the original codification, recoding the original data (or only a relevant part of it), or using more sophisticated space-time codification 4.

3. The number of required retransmissions to decode a packet. This value depends on:

a. The peer-to-peer channel gains between all the players in the communication, i.e., the source, the destination, and the helpers.

b. The forwarding technique exploited by the helpers.

c. The technique exploited by the destination device to combine the different retransmissions received from uncorrelated paths.

4. The MAC protocol used for the contention among the different helpers.

In the next section, we describe the specific challenges that an MC-ARQ scheme poses to the MAC sub-layer.

\section{MC-ARQ: Challenges at the MAC Layer}

The MC-ARQ scheme presented in this paper has some particularities that claim for a redesign of the traditional concept of MAC protocols. This transformation has to be done at three different levels; one at the cellular interface, one at the short-range network formed for the cooperation phase, and another one at the inter-technology ARQ scheme.

First, the MAC protocol executed in the cellular network should enable the overhearing of data transmissions. Typically, in cellular systems each user has some allocated resource, e.g., time slot, frequency band, coding sequence, etc. Therefore, it is necessary to redesign and rethink the access methods of these wide range networks so that at least some users can listen to all other transmissions thus enabling cooperation.

Second, MAC protocols for wireless networks have been traditionally designed on the basis of not only maximizing throughput and minimizing delay, but also achieving fairness among the contending users. In addition, MAC protocols are designed to attain maximum performance in stable conditions, without paying much attention at transitory effects of network start-up. However, in the considered MC-ARQ scheme, upon the initialization of the cooperation phase, the short-range network has the two following unique characteristics:

1. The local sub-network formed by the active helpers surrounding the node claiming for cooperation, i.e., the wireless grid, is suddenly set into saturation 
conditions whenever the cooperation phase is initiated. Upon the transmission of a CFC packet, all the active helpers have a data packet ready to transmit in order to assist the failed transmission. Therefore, the shortrange wireless grid operates in saturation conditions.

2. Fairness might not be a major issue. The main goal is to attempt to assist the failed transmission as fast as possible, minimizing the use of the radio resources for a single transmission.

These two characteristics determine the way MAC protocols should operate within the context of MC-ARQ schemes in wireless grids.

Finally, the redesign of MAC protocols goes beyond the contention among the different helpers in the short-range network and the overhearing at the cellular link. The integration of two different technologies should be accompanied by a joint design of the MAC protocol at both the cellular network and the short-range network. This is a completely unexplored research field. Just as a simple example, the cellular network should positively acknowledge data packets even when they have not been successfully decoded. This may have implications at higher layers of the protocol stack in the light of consistency of the transmitted data.

In order to exemplify how these peculiarities affect the MAC layer design, we present in this paper the PRCSMA protocol as a MAC protocol suitable for the wireless grid part, i.e., the short-range network. The protocol operation is based on the IEEE 802.11 MAC protocol [9], but with some modifications that are required for the sake of backward compatibility and to match the requirements of the MC-ARQ scheme. Similar modifications may be done in the MAC protocol of the 802.15.4 standard [10] in order to operate under the proposed scheme. Before presenting the operation of the protocol in Section 5, in Section 4 we review the state of the art in the design of MAC protocols for cooperative communications, putting the emphasis on the reasons why they are not suitable for the MC-ARQ scheme.

\section{Related Work}

Several cooperative MAC protocols have been recently proposed, see [11][17]. However, these are not designed to support the considered MC-ARQ scheme. In particular, in [11] two versions of the CoopMAC protocol are designed in the context of $802.11 \mathrm{~b}$ WLANs in order to solve the performance anomaly problem induced by the multi-rate capability of the Distributed Coordination Function (DCF) of the Standard [7]. Korakis et al. implemented the protocol in off-the-shelf WLAN interfaces using open source wireless drivers, as reported in [12]. The main contribution in [12] is the description of the overall implementation process and the limitations found when attempting to implement the protocol. These limitations are mainly due to the constraints 
imposed by the time sensitive tasks performed by wireless cards' firmware. In addition, the CoopMAC was adapted to wireless networks using directional antennas in [13]. On the other hand, both the Cooperative-MAC (CMAC) and Forward Error Correction CMAC (FCMAC) protocols were presented in [14] within the context of $802.11 \mathrm{e}$ networks to improve the performance and to ensure a certain Quality of Service. In [15], the Cooperative Diversity Medium Access with Collision Avoidance (CD-MACA) protocol is proposed within the context of wireless ad hoc networks operating over the CSMA with Collision Avoidance (CSMA/CA) protocol. Although the general idea of CD-MACA is interesting, the definition in [15] is quite general and several implementation details are not considered. From an energy-efficient perspective, another cooperative MAC protocol is also presented within the context of ad hoc networks in [16]. This proposal integrates cooperative diversity into two different wireless routing protocols by embedding a distributed cooperative MAC. In [17] a cooperative MAC protocol was presented within the context of a mesh network formed by an access point, a number of regular stations and one fixed wireless router (helper).

These protocols do not take into account the unique conditions of the MCARQ scheme discussed in the previous section, and thus a new field for research is open. We present in the next section the PRCSMA as a MAC protocol conceived to support the concept of C-ARQ and thus easily applicable to a MCARQ scheme.

\section{Persistent Relay Carrier Sensing Multiple Access (PRCSMA)}

The PRCSMA protocol was presented and analyzed in [7] and [8], respectively, with the primary design goal of enabling devices equipped with CSMA-based wireless interfaces to cooperate within their surrounding grid upon the occurrence of a transmission error. Although it was originally designed within the context of C-ARQ schemes, it can be applied for the coordination of the helpers within the short-range interface of a MC-ARQ scheme.

The protocol works as follows: whenever a data packet from a source is received with unrecoverable errors at destination, a cooperation phase can be initiated. A CFC packet is broadcast after sensing the channel idle for a Short Inter Frame Silence (SIFS) period. Regular data transmissions in IEEE 802.11 are done after a longer silence period (Distributed IFS, referred to as DIFS), and thus cooperation phases have higher priority than regular data traffic. The CFC packet invites all the devices to become active helpers as long as they meet some predefined helper selection criteria, which are not specified in the basic definition of PRCSMA. It is worth mentioning that although the optimal scheme would consist in selecting the best helper for each cooperation phase, the approach in PRCSMA is to select a set of the most appropriate active helpers in order to 
loosen the requirement of selecting exactly the best candidate in each moment. An interesting open line of research might be focused on assessing the trade-off between the costs of selecting the best helper and the time required to solve the contention among a set of selected helpers.

Upon the reception of the CFC packet, all the devices which become active helpers get ready to forward their cooperative information. The specific PHY forwarding strategies applied at the helpers and the reconstructing mechanism implemented at the destination are out of the scope of the basic definition of PRCSMA. Therefore, without loss of generality, the packet transmitted by any helper will be referred to as a cooperative packet. Accordingly, the active helpers will try to get access to the channel in order to persistently transmit their cooperative packets. To do so, the MAC rules specified in the Distributed Coordination Function (DCF) of the IEEE 802.11 standard are used, considering the two following modifications:

1. There is no expected ACK associated to each transmitted cooperation packet.

2. Since the sub-network formed by the helper set works in saturation conditions, i.e., all the helpers have a data packet ready to be transmitted, it is necessary to execute a backoff mechanism at the beginning of the cooperation phase in order to avoid a certain initial collision. Therefore, those active helpers which do not have an already set backoff counter (from a previous transmission attempt) set it up and initiate a random backoff period before attempting to transmit for the first time. On the other hand, those helpers which already have a non-zero backoff counter value keep the value upon the initialization of a cooperation phase.

A cooperation phase is ended whenever either the destination is able to decode the original data packet by properly combining the different cooperative packets received from the helper set or a certain maximum cooperation timeout has elapsed. In the former case, i.e., a successful cooperation phase, an ACK packet is transmitted by the destination. In the latter case, i.e. if the original packet could not be decoded, a negative ACK (NACK) is transmitted by the destination station. In any case, all the helpers pop-out the cooperative packet from their queue upon the end of a cooperation phase.

According to all this operation, three implementation issues should be considered:

1. The CFC packet can be implemented with a regular RTS packet but using one of the reserved control subtypes to distinguish the packet from a normal RTS, as already done in [12].

2. As long as there is at least one active helper, the persistent behavior of PRCSMA eliminates the probability that the destination does not receive the required amount of local retransmissions by pretending there are infinite devices trying to cooperate.

3. The active helpers could execute either the basic or the collision avoidance (with RTS/CTS handshake) access mode during a cooperation phase. On 
the one hand, as data bit rates become higher, it becomes more critical to reduce the overhead associated to the payload in order to avoid an unnecessary waste of the radio resources; therefore, it would be desirable to use the basic access mode. However, the collision avoidance mechanism acts as a protection against the hidden terminal problem, and thus it will be mandatory in multi-hop networks.

\section{Performance Evaluation}

In order to assess the performance of the protocol, link level simulations for a cellular network combined with a WLAN system have been carried out. Due to the complexity of simulating such a hybrid scenario, we have decided to implement an isolated layout in a custom-made MATLAB simulator. A group of wireless nodes, all within the transmission range of each other, are associated to a distant BS. We consider that they are all equipped with both cellular and WLAN interfaces. In order to focus on the MAC evaluation and to avoid obscuring the results with other parameters such as the channel impairments or network topology effects, we have considered a pessimistic worst-case scenario. The downlink between the BS and one predetermined and fixed destination device is in bad conditions and all data packets are received with unrecoverable errors. Therefore, this destination requests cooperative retransmissions from the wireless grid upon the reception of any original data packet from the BS. We consider a saturated condition where the BS always has at least one data packet (with a constant length of 1500 bytes) to transmit. The data and control transmission rates (for the ACK packets with a constant length of 34 bytes, as in the IEEE 802.11 [9]) from the BS to the destination user are both set to a constant value of $6 \mathrm{Mbps}$.

The short-range network surrounding the destination device is formed by 10 active helpers, all of them within the transmission range of each other. These helpers execute PRCSMA to get access to the channel. They use the basic access method of the protocol, i.e., without RTS/CTS handshake. We assume that the data rates between the helpers and the destination for data and control transmissions are 54 and $6 \mathrm{Mbps}$, respectively. The initial size of the contention window has been set to 32 . The throughput, defined as the number of error-free payload bits (including coding bits) transmitted per second, of the cellular interface is illustrated in Figure 5. The curves represent the throughput as a function of the number of retransmissions required to decode a data packet that has been received with unrecoverable errors. The throughput of three different retransmission schemes is represented:

1. The traditional ARQ curve represents the throughput of the cellular interface in the case that upon transmission error, retransmissions are requested from the BS. Retransmissions are performed one after another 
until the destination is able to decode the packet without errors and acknowledge its reception.

2. The MC-ARQ curve represents the saturation throughput of the cellular interface in the case that the MC-ARQ scheme with the PRCSMA protocol is executed. In this case, retransmissions are performed through the shortrange interface and thus data communication in the cellular link can continue without interruption.

3. The C-ARQ curve represents the saturation throughput of the cellular interface in the case that a C-ARQ scheme is executed within the cellular interface (also executing the PRCSMA at the MAC layer).

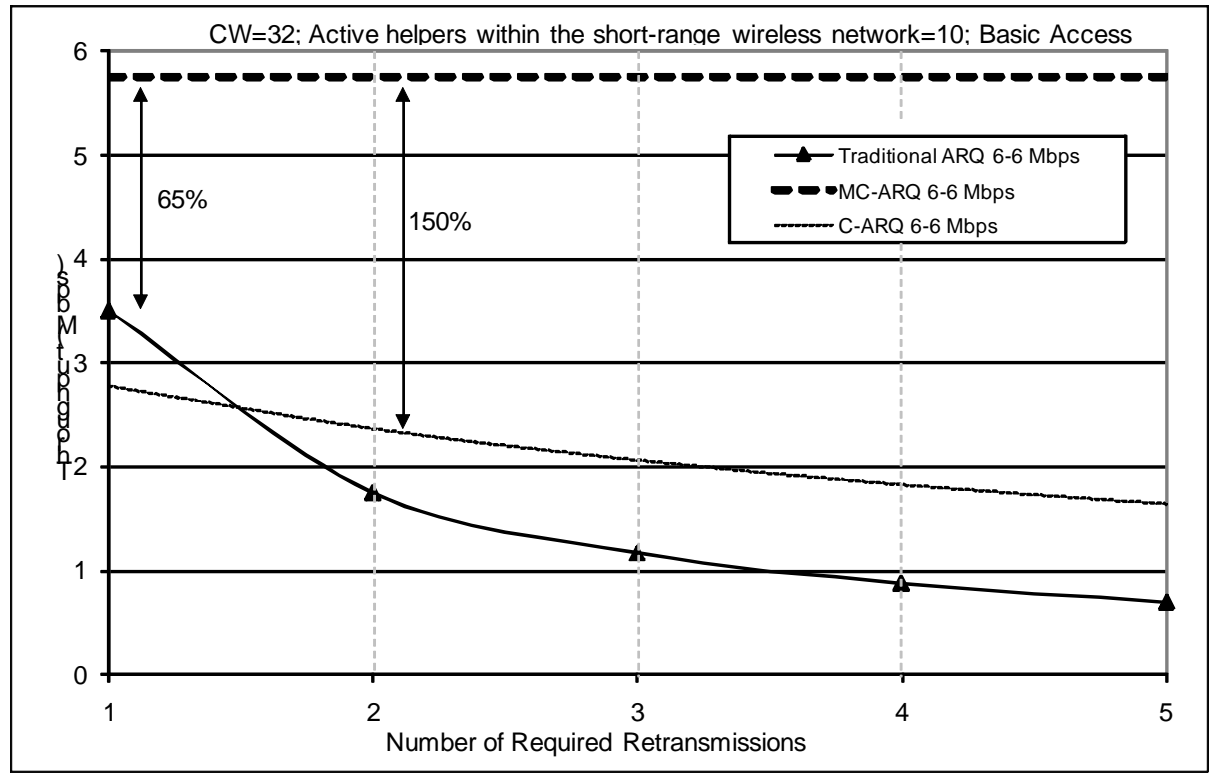

Figure $\mathbf{5}$ Throughput in the cellular network

It is possible to infer from Figure 5 that as the number of required retransmissions increases, the performance of the C-ARQ scheme outperforms the traditional non-cooperative ARQ scheme. Despite the extra overhead associated to the coordination among the helpers in the short-range network, the higher transmission rate between the helpers and the destination pays off the increased overhead. What results more interesting from the figure is that, if we eliminate retransmissions from the cellular interface, i.e., by means of the MCARQ scheme, then the throughput can reach the maximum theoretical performance of the network independently of the number of retransmissions needed. Under the considered PHY layer, this saturation throughput is of 5.7 Mbps (assuming that the only control information exchanged is composed of the ACK packets after each data packet transmission). Comparing this value with the 
curves from the C-ARQ and the traditional non-cooperative ARQ schemes, we see that the relative performance improvement between the different retransmission schemes becomes higher as the number of needed retransmissions grows. It seems reasonable to believe that this performance may be affected by the number of active helpers. To evaluate its impact, the average packet transmission delay of PRCSMA is represented in Figure 6 as a function of the number of active helpers within the grid, and for different number of required retransmissions $(K)$. This delay is defined as the time elapsed since the moment a packet is first eligible for transmission until $K$ retransmission from the relays are received. The curves show that given a number of required retransmissions, the average packet transmission delay remains almost independent of the number of active helpers. This is a key feature of the PRCSMA protocol which makes it suitable for this kind of communication schemes.

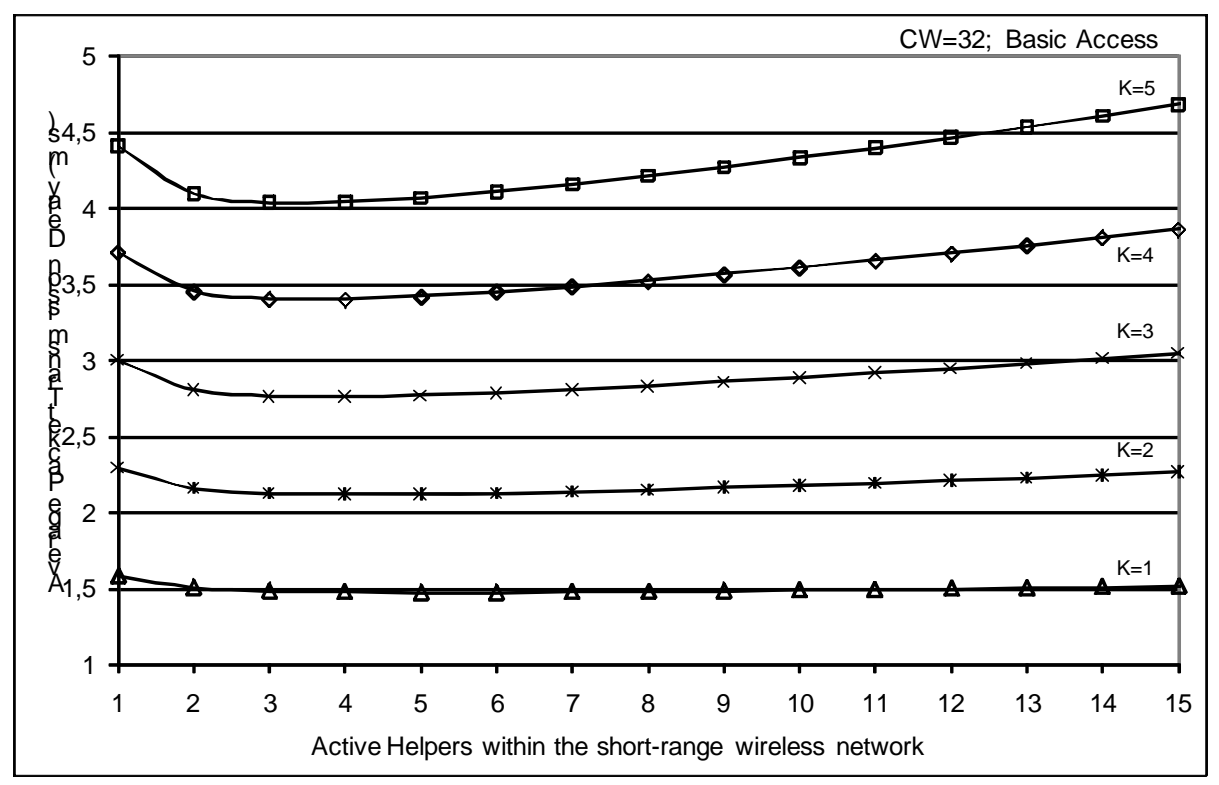

Figure 6 Average Packet Transmission Delay of PRCSMA for different number of retransmissions $(K)$

\section{Conclusions}

The increasing pervasive deployment of wireless devices equipped with several interfaces enables the rise of new communication paradigms. Wireless grids come up as a solution to integrate different wireless interfaces. In this paper we 
have presented Multi-Radio distributed Cooperative Automatic Retransmission Request (MC-ARQ) schemes as an approach to exploit the potential benefits of wireless grids. The main idea is that whenever a packet is received with errors within a cellular interface (infrastructure-based interface), retransmissions can be requested to neighboring stations which may have received an uncorrelated copy of the original transmission. These retransmissions can be performed through a short-range wireless interface reducing the occupation of the cellular links, improving the usage of the spectrum and reducing the overall average transmitted power.

In order to bring to life this promising concept it is still necessary to study it from different points of view. We have discussed in this paper the unique conditions that this kind of communication schemes poses to the MAC layer. In addition, we have identified PRCSMA as a MAC protocol suitable for the execution of the MC-ARQ scheme in a wireless network without degrading the performance of the cellular interface upon transmission error. Simulation results for a hybrid cellular/WLAN network are promising. In the worst-case example presented in this paper, the data throughput is improved by $65 \%$ when compared to traditional non-cooperative ARQ schemes if only one retransmission is required from the grid. This improvement factor grows as the number of required retransmissions increases. In this paper we have focused on the throughput performance. However, it remains as an interesting topic for research how the use of a MC-ARQ scheme may yield benefits in terms of reduced interference as well as energy consumption due to the lower transmission power required for the short-range transmissions. Therefore, the MC-ARQ scheme presented in this paper opens a wide range of interesting research topics to be covered in the upcoming years, and it constitutes a simple and practical approach to apply cooperation in real networks.

\section{Acknowledgment}

This work has been partially funded by the Research Projects NEWCOM++ (ICT216715), R2D2 (CP6-013), CENTENO (TEC2008-06817-C02-02), PERSEO (TEC2006-10459/TCM), COOLNESS (218163-FP7-PEOPLE-2007-3-1-IAPP) and by Generalitat de Catalunya (2009-SGR-940).

\section{References}

1. Cover, T. M. and Gamal, A. E., "Capacity Theorems for the Relay Channel," IEEE Transactions on Information Theory, vol. 25, no. 5, p. 572 (1979).

2. Sendonaris, A., Erkip, E. and Aazhang, B., "Station Cooperation Diversity-Part I: System Description," IEEE Transactions on Communications, vol. 51, no. 11, pp. 1927-1938 (2003). 
3. Laneman, J. N., Tse, D. N. C., and Wornell, G. W., "Cooperative Diversity in Wireless Networks: Efficient Protocols and Outage Behavior," IEEE Transactions on Information Theory, vol. 50, no. 12 (2004).

4. Fitzek, F. H. P., and Katz, M. D., "Cooperation in Wireless Networks: Principles and Applications," Springer (2006).

5. Fitzek, F. H. P., Katz, M., and Zhang, Q., "Cellular Controlled Short-Range Communication for Cooperative P2P Networking," in Wireless Research Forum (WWRF) 17, Heidelberg, Germany, WWRF, vol. WG 5, (2006).

6. Zimmermann, E., Herhold, P., and Fettweis, G., "The Impact of Cooperation on Diversity-Exploiting Protocols," in Proc. of the 59th IEEE Vehicular Technology Conference (2004)

7. Alonso-Zárate, J., Kartsakli, E., Verikoukis, C., and Alonso, L., "Persistent RCSMA: A MAC Protocol for a Distributed Cooperative ARQ Scheme in Wireless Networks," EURASIP Journal on Advanced Signal Processing, Special Issue on Wireless Cooperative Networks, vol. 2008, article ID 817401, pp. 13, (2008).

8. Alonso-Zárate, J., Alonso, L., and Verikoukis, Ch., "Performance Analysis of a Persistent Relay Carrier Sensing Multiple Access Protocol," IEEE Transactions on Wireless Communications, vol. 8, no. 12, (2009).

9. IEEE, Part 11: Wireless LAN Medium Access Control (MAC) and Physical Layer (PHY) Specifications, IEEE Std. 802.-11-99, (1999).

10. IEEE, Part 15.4: Wireless MAC and PHY layer specifications for low-rate wireless personal area networks," IEEE Std. 802.15.4-2006, (2006).

11. Liu, P., Tao, Z., Panwar, S., "CoopMAC: A Cooperative MAC for Wireless LANs," IEEE Journal on Selected Areas on Communications, vol. 25, no.2, (2007).

12. Korakis, T., Natayanan, S., Bagri, A., Panwar, S., "Implementing a Cooperative MAC Protocol for Wireless LAN," in Proc. of the IEEE International Conference on Communications (ICC’06), vol. 10, pp. 4805-4810 (2006).

13. Tao, Z., Korakis, T., Slutskiy, Y., Panwar, S., Tassiulas, L., "Cooperation and Directionality: A Coop-directional MAC for Wireless Ad Hoc Networks," in Proc. of the WiOpt (2007).

14. Shankar, S., Chou, C., Ghosh, M., "Cooperative Communication MAC (CMAC) A new MAC protocol for Next Generation Wireless LANs," in Proc. of the IEEE International Conference on Wireless Networks, Communications and Mobile Computing (2005).

15. Wang, X. and Yang, C., "A MAC Protocol Supporting Cooperative Diversity for Distributed Wireless Ad Hoc Networks," in Proc. of the IEEE International Symposium on PIMRC, Berlin, Germany, (2005).

16. Azgin, A., Altunbasak, Y., Alrebig, G., "Cooperative MAC and Routing Protocols for Wireless Ad Hoc Networks," in Proc. of the IEEE Globecom (2005).

17. Sadek, A., Ray Liu, K. J., Ephremides, A., "Collaborative Multiple-Access Protocols for Wireless Networks," in Proc. of the IEEE International Conference on Communications (ICC'06), (2006). 